\title{
Erratum to: Relationship of ground level enhancements with solar, interplanetary and geophysical parameters
}

\author{
K.A. Firoz • J. Hwang • I. Dorotovič • T. Pintér · \\ Subhash C. Kaushik
}

Received: 27 September 2010 / Accepted: 27 October 2010 / Published online: 17 November 2010

(C) Springer Science+Business Media B.V. 2010

\section{Erratum to: Astrophys Space Sci}

DOI 10.1007/s10509-010-0473-0

Equation (1) should read:

$I_{r}(\%)=\left(\frac{m \cdot I_{j}}{\sum_{k=1}^{m} I_{k}}-1\right) \times 100$

Reference "Whang 2007" in fifth paragraph of Sect. 1 should read "Wang 2007". The correct version of this reference is

Wang, R.: Large geomagnetic storms of extreme solar event periods in solar cycle 23. Adv. Space Res. 40, 1835-1841 (2007)

The last sentence in caption of Table 2 should be omitted. The caption of Table 2 should read as

The online version of the original article can be found under doi:10.1007/s10509-010-0473-0.

K.A. Firoz $(\bowtie) \cdot$ J. Hwang

Solar and Space Weather Research Group, Korea Astronomy and Space Science Institute, Yuseong-Gu, 305-348 Daejeon,

Republic of Korea

e-mail: kafiroz@kasi.re.kr

K.A. Firoz

e-mail: kazifiroz2002@gmail.com

I. Dorotovič · T. Pintér

Slovak Central Observatory, P.O. Box 42, 94701 Hurbanovo,

Slovak Republic

S.C. Kaushik

School of Physics, Jiwaji University, Gwalior, MP 474001, India
Table 2 Correlation coefficients of GLE peaks and overall CRI of each GLE with simultaneous geomagnetic indices ( $\mathrm{SymH})$, interplanetary magnetic field (IMF- $B_{\text {tot }}$ ), solar wind plasma velocity $\left(V_{\mathrm{sw}}\right)$, X-ray flux, and different energy channels of SEP fluxes. The correlations of GLE peaks $(n=6)$ with simultaneous solar, interplanetary and geophysical factors are examined. The data points $(n=6)$ of the GLE peak are taken for the rising part $(n=3)$ before peak point, peak point $(n=1)$ and descending after peak point $(n=2)$. The whole window of GLE event is taken as 24 hours that counted data number as $n=24 \times 12=288$ for 5 -minute resolution. The correlations of the overall CRI of the selected GLE events are examined for the whole time window $(n=288)$ 